\title{
Urinary Uromodulin/Creatinine Ratio as a Potential Clinical Biomarker for Chronic Kidney Disease Patients with Gout: A Pilot Study
}

\author{
Chien-Hsing Wu ${ }^{a}$ Chih-Chao Yang ${ }^{a}$ Hsueh-Wen Chang ${ }^{b}$ Bin Huang ${ }^{c, d}$ Chung-Jen Chen ${ }^{e}$ \\ Eton I-Cheng Lin ${ }^{f}$ Chien-Yi Wu ${ }^{g}$ Yueh-Hua Chung ${ }^{\text {h }}$ Yu-Han Hsu ${ }^{i}$ Chien-Te Lee ${ }^{a}$ \\ Feng-Rong Chuang ${ }^{\text {a }}$ \\ a Division of Nephrology, Kaohsiung Chang Gung Memorial Hospital and Chang Gung University College of \\ Medicine, Kaohsiung, Taiwan; 'bepartment of Biological Sciences, National Sun Yat-Sen University, Kaohsiung, \\ Taiwan; ' Department of Biomedical Science and Environmental Biology, College of Life Science, Kaohsiung Medical

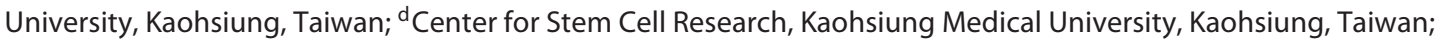 \\ e Division of General Internal Medicine, Kaohsiung Medical University Hospital, Kaohsiung, Taiwan; ${ }^{\mathrm{f}}$ Department \\ of Clinical Immunology and Allergy, The Royal Melbourne Hospital, Melbourne, VIC, Australia; ${ }^{9}$ Department of

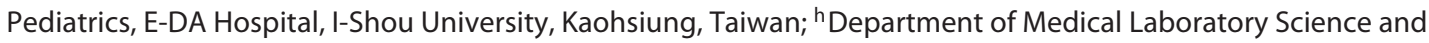 \\ Biotechnology, Kaohsiung Medical University, Kaohsiung, Taiwan; 'Department of Pharmacy, Kaohsiung Chang \\ Gung Memorial Hospital and Chang Gung University College of Medicine, Kaohsiung, Taiwan
}

\section{Significance of the Study}

- The study revealed that the excretion of urinary uromodulin is significantly decreased in chronic kidney disease (CKD) patients with gout. These findings indicate that uromodulin may have the potential to be used as a predictive marker for CKD in patients with gout.

\section{Keywords}

Uromodulin - Chronic kidney disease - Uric acid · Gout · Hyperuricemia

\section{Abstract \\ Objective: Recent studies have reported that reduced excre- tion of urinary uromodulin is associated with renal tubular function and risks of progressive kidney disease. Gouty ne- phropathy is usually seen in patients with gout. Patients with chronic gouty nephropathy are characterized by the deposi- tion of monosodium urate crystals primarily involving the collecting ducts in the medulla. We postulated that this cor- relation may be specific to gout and may serve as a useful}

\begin{tabular}{ll}
\hline KARGER & $\begin{array}{l}\text { ( 2019 The Author(s) Karger } \\
\text { Published by S. Karger AG, Basel }\end{array}$ \\
E-Mail karger@karger.com & $\begin{array}{l}\text { This is an Open Access article licensed under the Creative Commons } \\
\text { Attribution-NonCommercial-4.0 International License (CC BY-NC) } \\
\text { (http://www.karger.com/Services/OpenAccessLicense), applicable to } \\
\text { the online version of the article only. Usage and distribution for com- } \\
\text { mercial purposes requires written permission. }\end{array}$
\end{tabular}

biomarker for chronic kidney disease (CKD). Materials and Methods: A total of 114 Taiwanese patients diagnosed with gout $(n=72)$, CKD $(n=26)$, or healthy volunteers $(n=16)$ were prospectively enrolled for this study from the Rheumatology and Nephrology Outpatient Clinics of our institution. We obtained urine and blood samples on patient visits to the outpatient clinics. Demographic data were obtained from medical records. Results: In patients with gout, the spot urinary uromodulin/creatinine ratio (uUMCR; $\mathrm{mg} / \mathrm{g}$ ) in patients with CKD was significantly lower than that in those without CKD (CKD group: 2.2; non-CKD group: 5.6, $p=0.005)$. Multivariate analysis revealed that patients with CKD and gout had a lower UUMCR than those with gout alone $(p=0.028)$. A significant association was not observed in our non-gout 
cohort. Conclusion: The association of decreased uUMCR with CKD status was identified only in patients with gout in the present study. We believe that uUMCR might serve as an indicator of differential CKD in patients with gout.

(c) 2019 The Author(s)

Published by S. Karger AG, Basel

\section{Introduction}

Uromodulin (UMOD; also known as Tamm-Horsfall protein) is a glycoprotein produced in the tubular cells of the thick ascending limb (TAL) of the loop of Henle and in the early distal tubule and released into the tubular lumen. It is the most common protein found in the urine of healthy individuals. The exact role of UMOD is unclear, but it is likely to have a spectrum of functions in the renal tubules. This glycoprotein suppresses $T$ cells in vitro, prevents adhesion of uropathogenic Escherichia coli to the uroepithelium [1], and influences the homeostasis of electrolytes and the transporter function in the TAL [2], in addition to protecting from ischemic renal injury possibly via downregulation of toll-like receptor 4 [3]. Thus, the abundance of urinary UMOD (uUMOD) may reflect its apparent multimodal protective effects in the renal tubules, namely anti-inflammatory, anti-infective, and electrolyte-handling effects.

The association between UMOD and chronic kidney disease (CKD) has attracted considerable interest. Previous studies have shown that uUMOD is decreased in patients with CKD $[4,5]$. However, not all patients with $\mathrm{CKD}$ have decreased excretion of uUMOD as it has also been demonstrated that patients with $\mathrm{CKD}$ had a greater range of uUMOD excretion than normal subjects [6]. Furthermore, a recent study has shown that $22 \%$ of patients with CKD had normal uUMOD levels [7]. More recently, a case-control study reported an association between higher levels of uUMOD and the incidence of CKD [8]. Petzold et al. [9] demonstrated a lack of association of urinary UMOD and eGFR in patients with autosomal dominant polycystic kidney disease patients. Studies evaluating uUMOD levels with CKD prevalence and incidence have not yielded consistent results.

The potential role of hyperuricemia in reducing kidney function has become the subject of intense interest and speculation. Most studies have documented that elevated serum uric acid independently predicts the development of CKD [10-13]. Mild hyperuricemia, induced in normal rats by the uricase inhibitor (oxonic acid), induced oxidative stress and endothelial dysfunction, resulting in the development of both systemic and glomerular hypertension in association with elevated renal vascular resistance, reduced renal blood flow, and led to the development of arteriolosclerosis, glomerular injury, and tubulointerstitial fibrosis [14-16].

We have previously demonstrated that the spot $\mathrm{uUMOD} /$ creatinine ratio (uUMCR; $\mathrm{mg} / \mathrm{g}$ ) is significantly and positively correlated with eGFR in patients with gout [17]. We postulated that this correlation may be specific to gout. Here, we expanded our previous cohort to include a non-gout population, with and without CKD, for comparison. An important assumption was that patients with an established diagnosis of gout were more likely to have chronic urate-related renal damage, as supported by recent large-scale epidemiological studies [18, 19]. Leakage of UMOD has been known to cause inflammation of renal tissue, and a previous study [7] reported that damage to the TAL of a nephron and cells distal to the TAL results in leakage of UMOD into the interstitium, and subsequent inflammation. We hypothesized that the uUMOD decreased significantly compared to non-gout CKD, and this association was specific to patients with gout.

\section{Subjects and Methods}

\section{Participants}

Patients were prospectively recruited from the Rheumatology and Nephrology Outpatient Clinic at Kaohsiung Chang Gung Memorial Hospital between January 1, 2008 and May 31, 2012. Overall, 114 Taiwanese patients including patients diagnosed with gout with or without CKD $(n=72)$, CKD without gout $(n=26)$, and healthy volunteers $(n=16)$ were enrolled. Patients with gout were mainly recruited from the Rheumatology Outpatient Clinic and patients with CKD were recruited from the Nephrology Outpatient Clinic. The healthy volunteers were recruited from a health evaluation center.

The diagnosis of gout in these patients was confirmed by a rheumatologist and fulfilled the 1977 American College of Rheumatology's diagnostic criteria for gout [20]. The diagnosis and definition of CKD was based on the eGFR (in $\mathrm{mL} / \mathrm{min} / 1.73 \mathrm{~m}^{2}$ ), which was estimated from the CKD-EPI creatinine equation (2009) [21]: eGFR CKD-EPI eGFR $=141 \times \min \left(\mathrm{S}_{\mathrm{Cr}} / \kappa, 1\right)^{\alpha}$ $\operatorname{xmax}\left(\mathrm{S}_{\mathrm{Cr}} / \kappa, 1\right)^{-1.209} \times 0.993^{\text {Age }} \times 1.018$ (if female) $\times 1.159$ (if Black). The diagnosis required an eGFR CKD-EPI eGFR $<60 \mathrm{~mL} / \mathrm{min} /$ $1.73 \mathrm{~m}^{2}$ for at least 3 months. The inclusion criteria were as follows: age $>18$ years with stable gout activity without recent acute gouty attacks for $>3$ months before participation in the study. Stable renal function and proteinuria were defined as variability in serum creatinine levels and proteinuria of $<20 \%$ for over 3 months before participation in the study. Exclusion criteria included pregnancy, recent admission ( $<3$ months from the time of the most recent visit to the clinics), regular use of non-steroidal anti-inflammatory drugs, presence of a known heritable illness transmitted as an autosomal dominant trait, and a family history of CKD.

The 114 patients were subdivided into non-CKD and CKD groups. All participants provided written informed consent, and the Institutional Review Board of Kaohsiung Chang Gung Memo- 


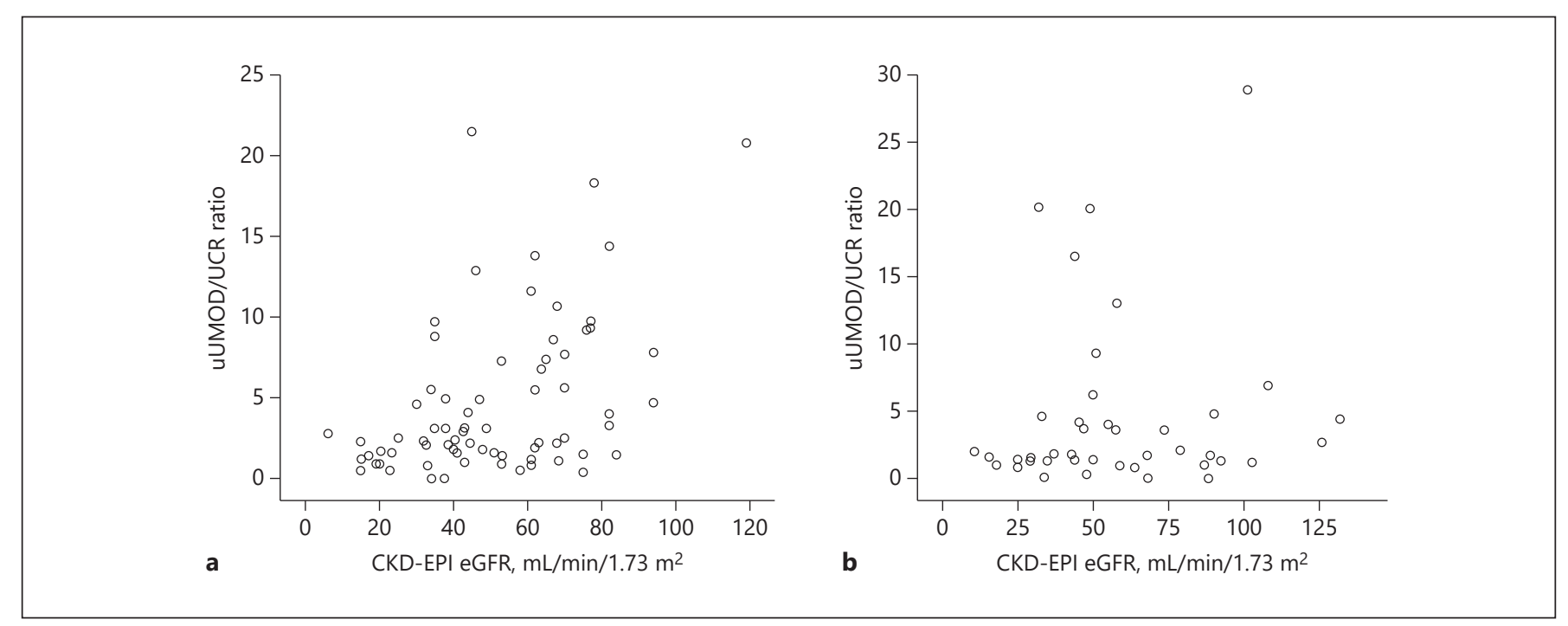

Fig. 1. Spearman's correlation of the uUMOD/UCR ratio $(\mathrm{mg} / \mathrm{g})$ and CKD-EPI eGFR in 72 patients with gout $(\gamma=0.408, p<0.001 ; \mathbf{a})$ and 42 patients without gout $(\gamma=0.080, p=0.614 ; \mathbf{b})$.

rial Hospital approved the study protocol. All patient-identifiable information was stored in locked cabinets and/or secure electronic servers. Urine and blood samples were obtained from the participants at the outpatient clinics and urinary creatinine (UCR), uUMOD, serum uric acid, and serum creatinine levels were measured. Demographic data, including diabetes mellitus (DM), hypertension status, and smoking history, were obtained from the medical records.

\section{Measurement of Urinary UMOD}

uUMOD was quantified by a sandwich ELISA (BioVendorLaboratorní medicína a.s., Czech Republic) according to the manufacturer's instructions, and uUMOD excretion was expressed as the uUMCR (mg/g).

\section{Calculation of the uUMOD/UCR Ratio to Determine Daily}

UMOD Excretion

Daily uUMOD excretion was expressed as the uUMOD/UCR ratio to correct for different levels of patient hydration.

\section{Statistical Analysis}

Statistical analysis was performed with PASW version 17 for Windows (SPSS Inc., Chicago, IL, USA). The relationship between eGFR and uUMCR was assessed by the Spearman's correlation coefficient. Baseline variables, including continuous and categorical data, were compared between the CKD and nonCKD groups. All results are expressed as the median (interquartile range; IQR) for continuous data and as percentages for categorical data. The Fisher exact test was used to compare categorical data between the CKD and non-CKD groups and the Mann-Whitney U test was used to compare continuous variables between the two groups. Significant variables (age, uric acid, uUMCR, hypertension, DM, and smoking status) were included in the logistic regression model. A $p$ value $<0.05$ was considered statistically significant. All tests were two-tailed.

Urinary Uromodulin in CKD Patients with Gout

\section{Results}

Correlation of uUMCR with CKD-EPI eGFR

$\left(\mathrm{mL} / \mathrm{min} / 1.73 \mathrm{~m}^{2}\right)$ in Patients with Gout

Spearman's correlation analysis was used to assess the relationship between uUMCR and CKD-EPI eGFR in all 72 patients with gout. Our results indicated that uUMCR $(\mathrm{mg} / \mathrm{g})$ was positively correlated with CKDEPI eGFR (correlation coefficient $[\gamma]=0.408, p<0.001$; Fig. 1a).

\section{Correlation between uUMCR and CKD-EPI eGFR in Patients without Gout}

Spearman's correlation analysis was used to assess the relationship between uUMCR (mg/g) and CKD-EPI eGFR in all 42 patients without gout (Fig. 1b). Our results indicated that uUMCR $(\mathrm{mg} / \mathrm{g})$ was not significantly positively correlated with CKD-EPI eGFR in all 42 patients without gout $(\gamma=0.080, p=0.614)$.

\section{Association of Measured Variables with Gout Patients and $C K D$}

Table 1 shows the clinical characteristics of gout patients with or without CKD. Age and UUMCR in the CKD group were significantly higher than those in the nonCKD group $(p<0.05)$. Other variables, such as sex, presence of hypertension and DM, and serum uric acid levels were not significantly different between patients with and without CKD. 
Table 1. Univariate analysis between CKD $\left(e G F R<60 \mathrm{~mL} / \mathrm{min} / 1.73 \mathrm{~m}^{2}\right)$ and non-CKD (eGFR $\geq 60 \mathrm{~mL} / \mathrm{min} /$ $1.73 \mathrm{~m}^{2}$ ) in the gout cohorts

\begin{tabular}{lcrr}
\hline & Non-CKD $(n=30)$ & CKD $(n=42)$ & $p$ value \\
\hline Sex & & & \\
$\quad$ Male & & & \\
$\quad$ Female & $28(93.3)$ & $6(85.7)$ & \\
Hypertension & $2(6.7)$ & $15(35.7)$ & 1 \\
$\quad$ Yes & $11(36.7)$ & $27(64.3)$ & \\
$\quad$ No & $19(63.3)$ & $11(23.5)$ & 0.245 \\
DM & & $31(76.5)$ & $<0.001$ \\
$\quad$ Yes & $4(13.3)$ & $64.0(50.8-73.5)$ & 0.207 \\
$\quad$ No & $26(86.7)$ & $8.4(6.7-9.6)$ & $<0.001$ \\
Age, years & $7.5(6.4-8.9)$ & $1.8(1.6-2.2)$ & $<0.001$ \\
Serum uric acid, $\mathrm{mg} / \mathrm{dL}$ & $1.2(1.1-1.3)$ & $63.3(25.1-46.1)$ & $<.005$ \\
Serum creatinine, $\mathrm{mg} / \mathrm{dL}$ & $72.4(63.8-83.2)$ & $2.2(1.2-3.4)$ & 0.005 \\
CKD-EPI eGFR, $\mathrm{mL} / \mathrm{min} / 1.73 \mathrm{~m}^{2}$ & $5.6(1.8-9.4)$ & & \\
UMOD/UCR ratio $(\mathrm{mg} / \mathrm{g})$ & & & \\
\end{tabular}

Data are presented as $n(\%)$ or the median (IQR). Continuous variables were analyzed by the Mann-Whitney $\mathrm{U}$ test and categorical data by the Fisher exact test.

Table 2. Univariate analysis between CKD (eGFR $\left.<60 \mathrm{~mL} / \mathrm{min} / 1.73 \mathrm{~m}^{2}\right)$ and non-CKD (eGFR $\geq 60 \mathrm{~mL} / \mathrm{min} /$ $1.73 \mathrm{~m}^{2}$ ) in the non-gout cohorts

\begin{tabular}{|c|c|c|c|}
\hline & Non-CKD $(n=16)$ & $\mathrm{CKD}(n=26)$ & $p$ value \\
\hline \multicolumn{4}{|l|}{ Sex } \\
\hline Male & $7(76.1)$ & $18(79.4)$ & \multirow{2}{*}{0.121} \\
\hline Female & $9(23.9)$ & $8(20.6)$ & \\
\hline \multicolumn{4}{|l|}{ Hypertension } \\
\hline Yes & $7(39.1)$ & $12(39.7)$ & \multirow{2}{*}{1} \\
\hline No & $9(60.9)$ & $14(60.3)$ & \\
\hline \multicolumn{4}{|l|}{$\mathrm{DM}$} \\
\hline Yes & $6(15.2)$ & $5(23.5)$ & \multirow{2}{*}{1} \\
\hline No & $10(84.8)$ & $21(76.5)$ & \\
\hline Age, years & $47.5(32.5-52.3)$ & $62.0(51.5-73.3)$ & $<0.001$ \\
\hline Serum uric acid, mg/dL & $5.9(4.6-7.2)$ & $7.9(5.7-9.3)$ & 0.009 \\
\hline Serum creatinine, $\mathrm{mg} / \mathrm{dL}$ & $0.83(0.79-0.94)$ & $1.7(1.5-2.2)$ & $<0.001$ \\
\hline CKD-EPI eGFR, $\mathrm{mL} / \mathrm{min} / 1.73 \mathrm{~m}^{2}$ & $88.8(75.0-102.4)$ & $43.5(29.3-50.0)$ & $<0.001$ \\
\hline UMOD/UCR ratio $(\mathrm{mg} / \mathrm{g})$ & $1.7(0.9-4.2)$ & $1.8(1.3-5.0)$ & 0.371 \\
\hline
\end{tabular}

Data are presented as $n(\%)$ or the median (IQR). Continuous variables were analyzed by the Mann-Whitney $\mathrm{U}$ test and categorical data the Fisher exact test.

\section{Association of Measured Variables with Non-Gout}

Patients and CKD

Table 2 shows the clinical characteristics of patients without gout, with and without CKD. Age and serum uric acid levels in the CKD group were significantly higher than those in the non-CKD group $(p<0.05)$. Other variables, such as sex, presence of hypertension and DM, and uUMCR, were not significantly different between patients with and without CKD. 
Table 3. Univariate analysis of the CKD-EPI eGFR $\left(\mathrm{mL} / \mathrm{min} / 1.73 \mathrm{~m}^{2}\right)$ and UMOD/UCR ratio $(\mathrm{mg} / \mathrm{g})$ between the non-gout and gout cohorts

\begin{tabular}{|c|c|c|c|c|c|c|c|c|c|}
\hline Patient characteristics & $\begin{array}{l}\text { Patients } \\
\text { with gout }\end{array}$ & $\begin{array}{l}\text { Patients } \\
\text { without gout }\end{array}$ & $p$ value & \multicolumn{2}{|l|}{ CKD } & $p$ value & \multicolumn{2}{|l|}{ Non-CKD } & $p$ value \\
\hline UMOD/UCR ratio & $\begin{array}{l}2.5 \\
(1.4-7.2)\end{array}$ & $\begin{array}{l}1.8 \\
(1.2-4.5)\end{array}$ & 0.163 & $\begin{array}{l}2.2 \\
(1.2-3.4)\end{array}$ & $\begin{array}{l}1.8 \\
(1.3-5.0)\end{array}$ & 0.767 & $\begin{array}{l}5.6 \\
(1.8-9.4)\end{array}$ & $\begin{array}{l}1.7 \\
(0.9-4.2)\end{array}$ & 0.012 \\
\hline
\end{tabular}

Data are presented as the median (IQR).

Table 4. Multivariate logistic regression analysis to explore the association of uUMCR with risk of CKD

\begin{tabular}{lcr}
\hline Factors & Odds ratio $(95 \% \mathrm{CI})$ & $p$ value \\
\hline Age (years) & $1.14(1.08-1.20)$ & $<0.001$ \\
Serum uric acid $(\mathrm{mg} / \mathrm{dL})$ & $1.89(1.30-2.76)$ & 0.001 \\
Gout (yes vs. no) & $0.73(0.16-3.26)$ & 0.684 \\
uUMOD/UCR $(\mathrm{mg} / \mathrm{g})$ & $1.01(0.88-1.15)$ & 0.882 \\
Gout (yes vs. no) $\times$ uUMOD/UCR $(\mathrm{mg} / \mathrm{g})$ & $0.80(0.66-0.98)$ & 0.028 \\
\hline
\end{tabular}

Association of uUMCR, Residual Renal Function, and Gout

Residual renal function and uUMCR were not significantly different between the patients with and without gout (Table 3).

\section{Association of Categorical and Continuous Variables with CKD}

Finally, we used logistic regression to determine whether serum uric acid levels, age, and uUMCR, hypertension, and DM were associated with CKD (Table 4). Our results indicate that higher uUMCR posed a significantly decreased risk of CKD in patients with gout. Elevated serum uric acid levels and older patients had statistically significant increased risks of CKD.

\section{Discussion}

This is the first study that demonstrates a positive correlation between urinary UMCR and eGFR in a population with gout, and non-correlation in a population without gout. Importantly, no significant difference was found in the proportion of individuals with classical renal risk factors between the gout and non-gout cohort, such as hypertension, diabetes, and baseline
eGFR. However, the level of UMCR in patients with CKD did not differ significantly between patients with and without gout (Table 3 ). This does not disprove the utility of uUMCR in CKD because the presence of gout is more likely to represent a greater risk rather than an absolute reflection of urate-related renal damage. Clinically, patients with previously diagnosed gout may have optimally controlled serum uric acid levels and minimal urate renal damage; conversely, patients without a prior diagnosis of gout may have significant hyperuricemia and marked urate-related renal damage. This is a limitation of our study, and may explain the lack of difference in UMOD excretion in the CKD sample. However, the level of UMCR in the non-CKD cohort was significantly higher in patients with gout (Table 3). This suggests that uUMCR may be used as a screening tool to detect early urate-related renal damage in patients with gout.

A challenge to the use of urinary UMCR as a biomarker is the potential lack of an absolute "normal" value. The result in the gout cohort (Fig. 1) suggests that different renal functions may have different "normal" urinary UMCR. Further longitudinal studies are essential to delineate its clinical utility.

A major cause of hyperuricemia is underexcretion of urate by the kidneys, but sufficient evidence also exists to 
define hyperuricemia as the cause of worsening renal function. In an interventional study, Whelton et al. [22] predicted that $1.15 \mathrm{~mL}$ of eGFR would be preserved for every $1 \mathrm{mg} / \mathrm{dL}$ reduction of serum uric acid in patients with gout. It is therefore pertinent that future clinical interventional studies examining hyperuricemia and renal function should include serum uric acid as an important parameter.

\section{Non-Correlation of Serum Urate Level with uUMCR}

This study indicated uUMCR as a potential biomarker for urate-related renal damage. In addition, the results suggested that serum uric acid is a stronger predictor of CKD than uUMCR. However, we believe these two parameters reveal separate aspects of urate-related renal dysfunction. Serum uric acid reflects the total urate load that the kidney has to process; the higher the level, the more likely it is that the nephrons are damaged. The excretion of uUMCR may suggest the degree of urate-related damage to the TAL of Henle, where UMOD is exclusively produced. Therefore, the relationship between serum uric acid and uUMCR is perhaps comparable to that of serum glucose and glycosylated hemoglobin. Similar to serum glucose, the serum urate level is known to have significant diurnal variation and daily fluctuation [2325 ] and is heavily influenced by diet. Although serial uric acid sampling may better reveal the chronicity of the urate load, an "ideal" target serum uric acid level may not be sufficient in guiding urate-lowering therapy for uraterelated renal damage. Different individuals may likely have different thresholds for urate-related renal damage, based on their renal function and metabolism; this threshold may be reflected through the uUMCR.

Two recent large-scale observational studies show that the $\mathrm{uUMOD}$ is an indicator of tubular health and could be used to identify individuals at higher risk of progressive kidney disease and mortality $[26,27]$. Urate crystal nephropathies are common in patients with gout, with frequencies of 79-99\% based on autopsy studies [10]. Tubular damage in patients with gout may be worse in CKD compared to non-CKD patients. Future studies are needed to confirm the results and whether it plays a causal role in actual tubular health in patients with gout and CKD.

\section{Conclusion}

In this study, we have reported a subset of patients with $\mathrm{CKD}$ and gout who are associated with a lower excretion of $\mathrm{uUMOD}$ which is absent in patients without gout. We postulate that, prior to any clinically significant renal impairment, uUMCR may have the potential to be used as an indicator for CKD in patients with gout.

\section{Acknowledgments}

We appreciate the Biostatistics Center, Kaohsiung Chang Gung Memorial Hospital for the statistical work. We thank our patients for contributing samples for this study. This study was supported by a grant from Chang Gung Memorial Hospital (grant No. CMRPG8B0251).

\section{Statement of Ethics}

All participants provided written informed consent, and the Institutional Review Board of Kaohsiung Chang Gung Memorial Hospital approved the study protocol.

\section{Disclosure Statement}

The authors declare that they have no conflicts of interests regarding the publication of this paper.

\section{References}

1 Pak J, Pu Y, Zhang ZT, Hasty DL, Wu XR. Tamm-Horsfall protein binds to type 1 fimbriated Escherichia coli and prevents E. coli from binding to uroplakin Ia and Ib receptors. J Biol Chem. 2001 Mar;276(13):9924-30.

2 Bachmann S, Mutig K, Bates J, Welker P, Geist B, Gross V, et al. Renal effects of TammHorsfall protein (uromodulin) deficiency in mice. Am J Physiol Renal Physiol. 2005 Mar; 288(3):F559-67.
3 El-Achkar TM, Wu XR, Rauchman M, McCracken R, Kiefer S, Dagher PC. TammHorsfall protein protects the kidney from ischemic injury by decreasing inflammation and altering TLR4 expression. Am J Physiol Renal Physiol. 2008 Aug;295(2):F534-44.

4 Gudbjartsson DF, Holm H, Indridason OS, Thorleifsson G, Edvardsson V, Sulem P, et al. Association of variants at UMOD with chronic kidney disease and kidney stones - role of age and comorbid diseases. PLoS Genet. 2010 Jul;6(7):e1001039.
5 Chakraborty J, Below AA, Solaiman D. Tamm-Horsfall protein in patients with kidney damage and diabetes. Urol Res. 2004 May; 32(2):79-83.

6 Thornley C, Dawnay A, Cattell WR. Human Tamm-Horsfall glycoprotein: urinary and plasma levels in normal subjects and patients with renal disease determined by a fully validated radioimmunoassay. Clin Sci (Lond). 1985 May;68(5):529-35. 
7 Prajczer S, Heidenreich U, Pfaller W, Kotanko P, Lhotta K, Jennings P. Evidence for a role of uromodulin in chronic kidney disease progression. Nephrol Dial Transplant. 2010 Jun;25(6):1896-903.

8 Köttgen A, Pattaro C, Böger CA, Fuchsberger C, Olden M, Glazer NL, et al. New loci associated with kidney function and chronic kidney disease. Nat Genet. 2010 May;42(5):376-84.

9 Petzold K, Poster D, Krauer F, Spanaus K, Andreisek G, Nguyen-Kim TD, et al. Urinary biomarkers at early ADPKD disease stage. PLoS One. 2015 Apr;10(4):e0123555.

10 Kang DH, Nakagawa T. Uric acid and chronic renal disease: possible implication of hyperuricemia on progression of renal disease. Semin Nephrol. 2005 Jan;25(1):43-9.

11 Johnson RJ, Nakagawa T, Jalal D, SánchezLozada LG, Kang DH, Ritz E. Uric acid and chronic kidney disease: which is chasing which? Nephrol Dial Transplant. 2013 Sep; 28(9):2221-8.

12 Zhu P, Liu Y, Han L, Xu G, Ran JM. Serum uric acid is associated with incident chronic kidney disease in middle-aged populations: a meta-analysis of 15 cohort studies. PLoS One. 2014 Jun;9(6):e100801.

13 Sipahi S, Acikgoz SB, Genc AB, Yildirim M, Solak Y, Tamer A. The Association of Vitamin D Status and Vitamin D Replacement Therapy with Glycemic Control, Serum Uric Acid Levels, and Microalbuminuria in $\mathrm{Pa}$ tients with Type 2 Diabetes and Chronic Kidney Disease. Med Princ Pract. 2017;26(2): 146-51.
14 Mazzali M, Kanellis J, Han L, Feng L, Xia YY, Chen Q, et al. Hyperuricemia induces a primary renal arteriolopathy in rats by a blood pressure-independent mechanism. Am J Physiol Renal Physiol. 2002 Jun;282(6):F9917.

15 Kang DH, Nakagawa T, Feng L, Watanabe S, Han L, Mazzali M, et al. A role for uric acid in the progression of renal disease. J Am Soc Nephrol. 2002 Dec;13(12):2888-97.

16 Sánchez-Lozada LG, Tapia E, Santamaría J, Avila-Casado C, Soto V, Nepomuceno T, et al. Mild hyperuricemia induces vasoconstriction and maintains glomerular hypertension in normal and remnant kidney rats. Kidney Int. 2005 Jan;67(1):237-47.

$17 \mathrm{Wu} \mathrm{CH}$, Lee CT, Lee $\mathrm{CH}$, Cheng TT, Chang $\mathrm{HW}$, Lin E, et al. Urinary UMOD excretion and chronic kidney disease in gout patients: cross-sectional case-control study. Ren Fail. 2011;33(2):164-8.

$18 \mathrm{Yu} \mathrm{KH}$, Kuo CF, Luo SF, See LC, Chou IJ, Chang HC, et al. Risk of end-stage renal disease associated with gout: a nationwide population study. Arthritis Res Ther. 2012 Apr; 14(2):R83.

19 Nickeleit V, Mihatsch MJ. Uric acid nephropathy and end-stage renal disease-review of a non-disease. Nephrol Dial Transplant. 1997 Sep;12(9):1832-8.

20 Wallace SL, Robinson H, Masi AT, Decker JL, McCarty DJ, Yü TF. Preliminary criteria for the classification of the acute arthritis of primary gout. Arthritis Rheum. 1977 Apr;20(3): 895-900.
21 Levey AS, Stevens LA. Estimating GFR using the CKD Epidemiology Collaboration (CKDEPI) creatinine equation: more accurate GFR estimates, lower CKD prevalence estimates, and better risk predictions. Am J Kidney Dis. 2010 Apr;55(4):622-7.

22 Whelton A, MacDonald PA, Chefo S, Gunawardhana L. Preservation of renal function during gout treatment with febuxostat: a quantitative study. Postgrad Med. 2013 Jan; 125(1):106-14.

23 Devgun MS, Dhillon HS. Importance of diurnal variations on clinical value and interpretation of serum urate measurements. J Clin Pathol. 1992 Feb;45(2):110-3.

24 Goldstein RA, Becker KL, Moore CF. Serum urate in healthy men. Intermittent elevations and seasonal effect. N Engl J Med. 1972 Sep; 287(13):649-50.

25 Zachau-Christiansen B. The variations in serum uric acid during 24 hours and from day to day. Scand J Clin Lab Invest. 1957;9(3): 244-8.

26 Pruijm M, Ponte B, Ackermann D, Paccaud F, Guessous I, Ehret G, et al. Associations of Urinary Uromodulin with Clinical Characteristics and Markers of Tubular Function in the General Population. Clin J Am Soc Nephrol. 2016 Jan;11(1):70-80.

27 Garimella PS, Biggs ML, Katz R, Ix JH, Bennett MR, Devarajan P, et al. Urinary uromodulin, kidney function, and cardiovascular disease in elderly adults. Kidney Int. 2015 Nov; 88(5):1126-34. 